\title{
CRIPTOCOCOSE PULMONAR: ASPECTOS NA TOMOGRAFIA COMPUTADORIZADA*
}

\author{
Ana Carina Gamboa da Silva ${ }^{1}$, Edson Marchiori ${ }^{2}$, Arthur Soares Souza Jr. ${ }^{3}$, Klaus L. Irion ${ }^{4}$
}

Resumo A criptococose pulmonar é uma doença causada pelo Criptococcus neoformans, um fungo unimórfico que possui distribuição mundial, existindo na mesma forma tanto no seu habitat natural quanto em animais e humanos. A doença possui apresentações clínica e patológica variáveis e pode manifestar-se tanto em pacientes com a imunidade normal como em imunocomprometidos, que representam a maioria dos casos. Neste trabalho são analisados os aspectos encontrados nas tomografias computadorizadas do tórax de 14 pacientes com criptococose pulmonar confirmada. Os achados mais freqüentes na tomografia do tórax foram as massas e os nódulos pulmonares. Outros aspectos observados foram as áreas de escavação, as consolidações, o espessamento do interstício peribroncovascular e o reticulado difuso. Massa pulmonar foi o achado isolado mais comum $(64,2 \%)$, seguido dos nódulos isolados ou múltiplos $(35,7 \%)$. Doença pulmonar difusa foi vista em apenas $14,2 \%$ dos casos. Os lobos superiores foram os mais comprometidos, sendo a doença mais comum nas regiões anteriores. A tomografia do tórax permitiu avaliar com precisão o grau de comprometimento do parênquima pulmonar.

Unitermos: Radiologia; Criptococose pulmonar; Tomografia computadorizada.

Abstract Pulmonary cryptococcosis: computed tomography findings.

Pulmonary cryptococcosis is caused by Cryptococcus neoformans, a world-wide distribution unimorphic fungus that exhibits the same form in its natural habitat, animals and humans. The disease presents variable clinical and pathologic features and occurs in immunocompetent and immunocompromised patients. We reviewed the computed tomography findings of 14 patients with confirmed pulmonary cryptococcosis. The most frequent computed tomography findings were masses and pulmonary nodules. Other abnormalities included cavities, areas of consolidation, peribronchovascular interstitial thickening and diffuse linear opacities. Pulmonary masses were the most frequent findings $(64.2 \%)$, followed by nodules $(35.7 \%)$ whereas diffuse pulmonary disease accounted for $14.2 \%$ of the cases. The most affected areas were the upper lobes and the anterior regions. Computed tomography scan of the chest allows a precise assessment of pulmonary parenchymal involvement in patients with pulmonary cryptococcosis.

Key words: Radiology; Pulmonary cryptococcosis; Computed tomography.

\section{INTRODUÇÃO}

A criptococose pulmonar é uma doença causada pelo Criptococcus neoformans, um fungo unimórfico que possui distribuição mundial, existindo na mesma forma

* Trabalho realizado no Departamento de Radiologia da Universidade Federal Fluminense (UFF), Niterói, RJ, no Senviço de Radiologia do Hospital Universitário Clementino Fraga Filho (HUCFF) da Universidade Federal do Rio de Janeiro (UFRJ), Rio de Janeiro, RJ, e no Hospital de Base da Faculdade de Medicina de São José do Rio Preto (Famerp), São José do Rio Preto, SP.

1. Médica Mestranda do Departamento de Radiologia da UFR.

2. Professor Titular do Departamento de Radiologia da UFF Coordenador Adjunto do Curso de Pós-Graduação em Radiologia da UFRJ.

3. Professor Adjunto da Famerp, Membro do Ultra X São José do Rio Preto.

4. Radiologista do Pavilhão Pereira Filho, Doutor em Medicina pela Universidade Federal do Rio Grande do Sul.

Endereço para correspondência: Prof. Dr. Edson Marchiori. Rua Thomaz Cameron, 438, Valparaíso. Petrópolis, RJ, 25685-120. E-mail: edmarchiori@zipmail.com.br

Recebido para publicação em 20/2/2003. Aceito, após revisão, em 20/3/2003. tanto no seu habitat natural quanto em animais e humanos ${ }^{(\mathbf{1})}$.

A doença possui apresentações clínica e patológica variáveis ${ }^{(2)}$ e pode manifestarse tanto em pacientes com a imunidade normal como em pacientes imunocomprometidos, que representam a maioria dos ca$\operatorname{sos}^{(3)}$. Fatores predisponentes para a criptococose incluem síndrome da imunodeficiência adquirida (SIDA), neoplasias hematológicas, transplante de órgão, terapia com corticóides, sarcoidose e doença de Hodgkin, dentre outras condições que comprometem a imunidade celular ${ }^{(\mathbf{4 , 5})}$. Todas estas condições devem ser previamente definidas, pois influenciam na morbidade e mortalidade dos pacientes, assim como as condições clínicas, laboratoriais e histopatológicas ${ }^{(\mathbf{6})}$.

Os hospedeiros comprometidos apresentam uma ampla variedade de anormalidades radiológicas, tais como nódulos simples, nódulos múltiplos que evoluem para confluência ou escavação, consolidação segmentar, broncopneumonia bilateral ou padrão misto, derrame pleural e linfonodomegalias. Os pacientes com integridade da imunidade apresentam maior tendência à formação de nódulo, sem predileção $\operatorname{lobar}^{(7)}$.

Neste trabalho foi realizado um estudo retrospectivo a partir das tomografias computadorizadas de tórax de 14 pacientes, provenientes de várias instituições médicas, objetivando demonstrar os aspectos tomográficos da criptococose pulmonar. A tomografia computadorizada representa uma importante etapa no diagnóstico desta doença, pois a partir deste exame é possível estabelecer uma correlação anátomoclínica, evidenciar o grau de acometimento do parênquima pulmonar, a extensão da infecção (pleural e mediastinal) e a presença de linfonodomegalias. 


\section{MATERIAIS E MÉTODOS}

Foi feito estudo retrospectivo das tomografias computadorizadas de tórax de 14 pacientes com criptococose pulmonar confirmada por estudos citológicos e histopatológicos, no período de setembro/1993 a novembro/2001. Os pacientes eram provenientes de seis diferentes instituições médicas do Rio de Janeiro, RJ, Porto Alegre, RS, e São José do Rio Preto, SP.

O diagnóstico de criptococose pulmonar foi confirmado pela presença do fungo Cryptococcus neoformans no tecido pulmonar, obtido por exame endoscópico com lavado, escovado e biópsia transbrônquica em 12 pacientes e biópsia a céu aberto em dois pacientes.

Não houve padronização nas rotinas do estudo tomográfico dos pacientes, já que eles procediam de instituições diferentes, sendo estudados de acordo com as rotinas de cada serviço.

As tomografias dos pacientes foram analisadas em filmes com janelas adequadas para avaliação do parênquima pulmonar e de mediastino.

Os objetivos da análise das tomografias computadorizadas foram:

a) localização das lesões;

b) distribuição unilateral ou bilateral das lesões;

c) apresentação nodular solitária ou múltipla;

d) presença de consolidação segmentar ou massas infiltrativas; e) presença de linfonodomegalias hilares e/ou mediastinais;

f) presença de derrame pleural;

g) presença de infiltrado reticular ou nodular.

\section{RESULTADOS}

\section{A - Aspectos clínicos}

Sete pacientes eram do sexo masculino $(50 \%)$ e sete, do sexo feminino (50\%), com idades variando de 28 a 62 anos e média de idade de 40 anos.

De maneira geral, os pacientes apresentaram sintomas respiratórios muito semelhantes entre si, tais como tosse, dor torácica, febre e escarros hemoptóicos.

A presença de doença disseminada foi evidente em seis pacientes $(42,8 \%)$, havendo concomitância do comprometimento pulmonar e neurológico. Dois pacientes $(14,2 \%)$ apresentavam doenças previamente conhecidas ao quadro de criptococose pulmonar (diabetes mellitus e hipotireoidismo associado a hepatopatia alcoólica e doença pulmonar obstrutiva crônica). Apenas um paciente era imunodeprimido, com o diagnóstico de SIDA estabelecido por ocasião da infecção pelo fungo. Treze pacientes eram imunocompetentes e não apresentavam fatores predisponentes para a infecção fúngica.

\section{B - Aspectos tomográficos}

As principais alterações encontradas nas tomografias computadorizadas de tó- rax dos 14 pacientes estudados foram as massas pulmonares, os nódulos, a escavação, o infiltrado intersticial reticular, as consolidações e o espessamento de septos interlobulares. A Tabela 1 evidencia a freqüência de cada um destes achados.

Tabela 1 Freqüência dos achados na tomografia computadorizada, nos 14 casos estudados.

\begin{tabular}{|l|c|c|}
\hline \multicolumn{1}{|c|}{ Achados } & $\mathrm{N}$ & $\%$ \\
\hline Massa pulmonar & 9 & $64,2 \%$ \\
- Contato com a pleura & 7 & $50,0 \%$ \\
- Escavação & 2 & $14,2 \%$ \\
Nódulo único & 2 & $14,2 \%$ \\
Nódulos múltiplos & 3 & $21,4 \%$ \\
Infiltrado reticular & 1 & $7,1 \%$ \\
Consolidações esparsas & 2 & $14,2 \%$ \\
Espessamento septal & 1 & $7,1 \%$ \\
Espessamento do interstício & 1 & $7,1 \%$ \\
\hline
\end{tabular}

Massa pulmonar foi a manifestação tomográfica mais freqüente, encontrada em nove dos 14 pacientes $(64,2 \%)$ (Figuras 1 , 2 e 3). As lesões configurando massa mostraram-se com bordas bem definidas e contornos irregulares nos nove casos estudados. O íntimo contato com a superfície pleural foi um achado marcante, presente em sete dos nove pacientes que apresentavam massa $(77,7 \%)$ (Figura 3). Escavação foi achado pouco freqüente, presente em apenas dois dos nove pacientes que cursaram com massa (22,2\%) (Figuras 1 e 2).

Os nódulos pulmonares foram o segundo achado mais comum, observados em

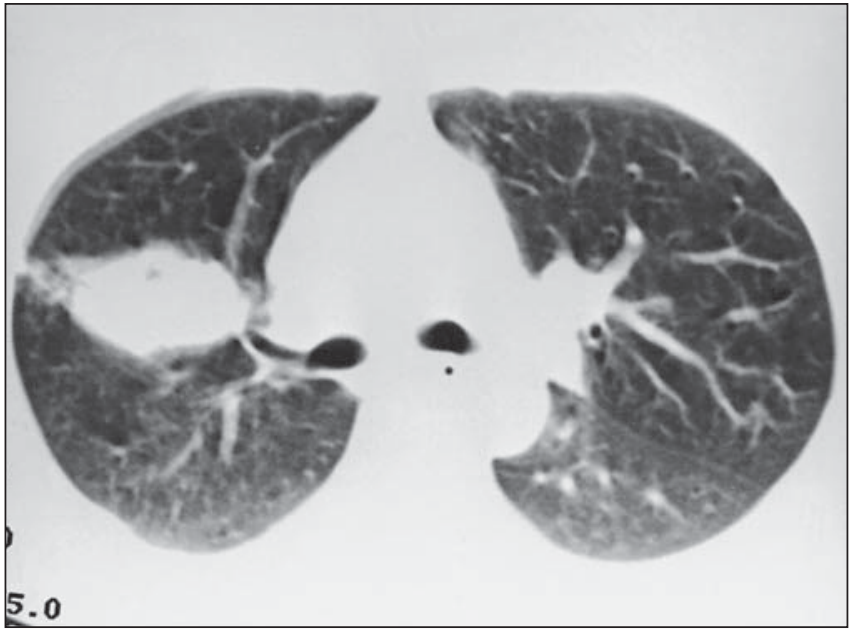

A

Figura 1. Tomografia computadorizada de tórax com janela para parênquima pulmonar demonstrando massa pulmonar com área hipodensa no interior. Em $\mathbf{B}$ observa-se melhor a presença de área escavada no interior da massa pulmonar.

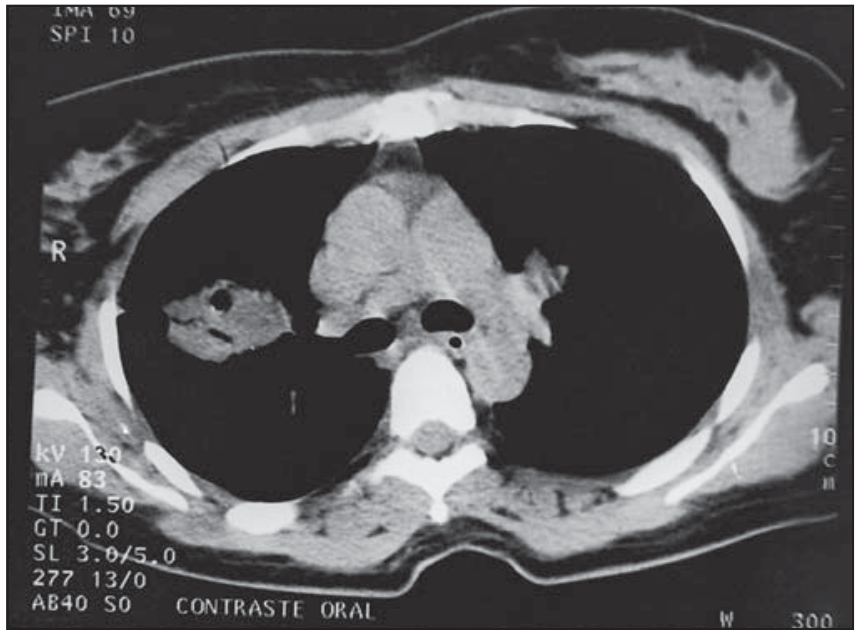

B 
Figura 2. Em A, tomografia computadorizada de tórax com janela para parênquima pulmonar demonstrando massa pulmonar, heterogênea, além de condensações parenquimatosas em contato com a superfície pleural, no lobo superior esquerdo. Em B e C, tomografia computadorizada de tórax com janela para mediastino evidenciando a massa pulmonar com área de escavação (C).
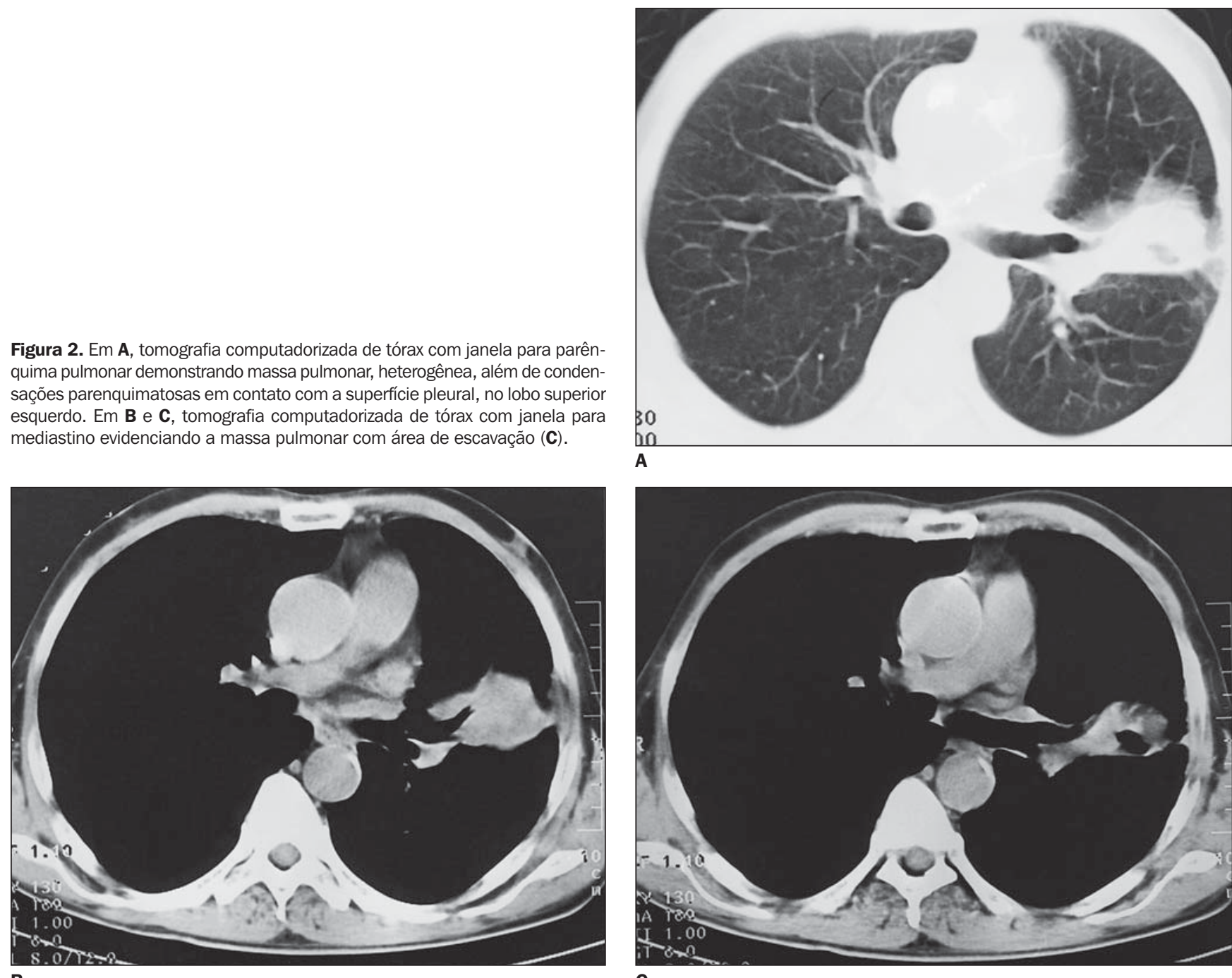

A

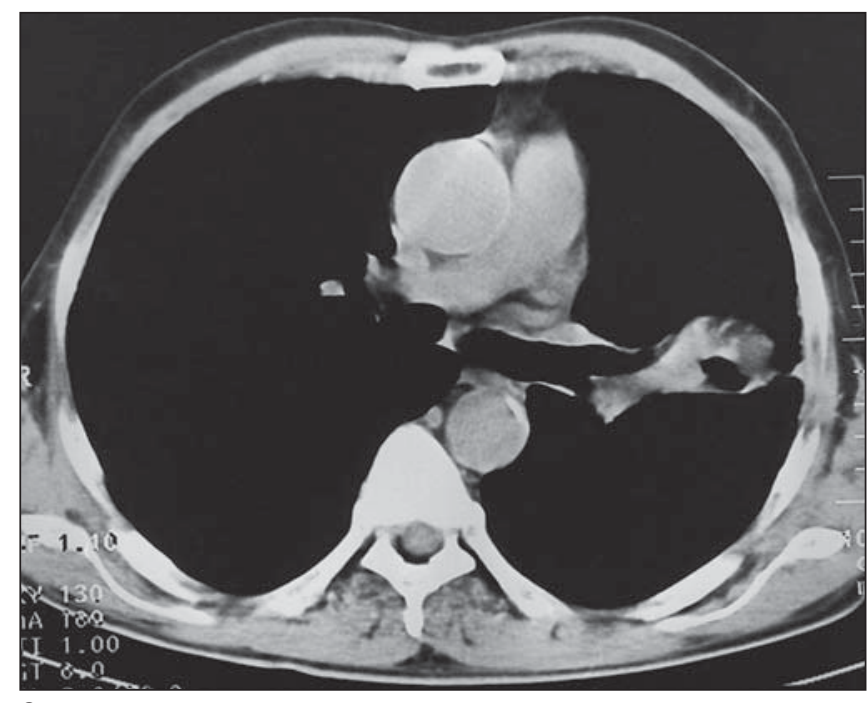

C

cinco casos $(35,7 \%)$, também se mostrando com bordas definidas e contornos irregulares. Em dois casos os nódulos eram únicos (40\%) (Figura 4). Um dos pacientes desenvolveu nódulos múltiplos, esparsos pelos pulmões, outro paciente apresentou nódulo associado à presença de infiltrado intersticial reticular (Figura 5), e em um outro caso houve associação com massa pulmonar.

Doença pulmonar difusa foi encontrada em dois pacientes (14,2\%). Em um caso foram observadas áreas de consolidação esparsas, além de espessamento dos septos interlobulares e do interstício peribroncovascular (Figura 6), e em outro foram constatados infiltrado intersticial com reticulações difusas pelo parênquima pulmonar, áreas de consolidação e formação nodular (Figura 5).
O comprometimento pulmonar foi unilateral em 11 dos 14 pacientes deste estudo $(78,5 \%)$ e bilateral em três casos $(21,4 \%)$. O pulmão direito foi acometido isoladamente em sete pacientes $(50 \%)$ e o esquerdo, em quatro $(28,5 \%)$.

Quanto à localização, houve predomínio das regiões anteriores, observado em seis pacientes $(42,8 \%)$; as regiões posteriores foram acometidas isoladamente em quatro casos (28,5\%). Quatro pacientes apresentaram envolvimento de ambas as regiões $(28,5 \%)$.

Avaliando a distribuição lobar da doença, observamos que cinco pacientes apresentaram envolvimento isolado do lobo superior $(35,7 \%)$; o lobo médio foi isoladamente afetado em três casos $(21,4 \%)$ e o lobo inferior, em apenas dois $(14,2 \%)$. O lobo superior e o inferior mostraram-se conjuntamente envolvidos em dois pacientes $(14,2 \%)$. A forma difusa da criptococose pulmonar foi observada em dois pacientes $(14,2 \%)$.

Derrame pleural ou linfonodomegalias hilares e/ou mediastinais não foram evidenciados nesta casuística.

\section{DISCUSSÃO}

No presente estudo observou-se que a criptococose pulmonar acometeu pacientes na faixa etária entre 24 e 62 anos, com média de idade de 40 anos, dados estes semelhantes aos encontrados por Rozenbaum e Gonçalves ${ }^{(\mathbf{8})}$, Chechani e Kamholz $^{(2)}$, Miller e Edelman ${ }^{(9)}$ e Roebuck et $a l .{ }^{(10)}$. Já Kerkering et al. ${ }^{(11)}$ referem um predomínio em pacientes com faixa etária mais avançada. 


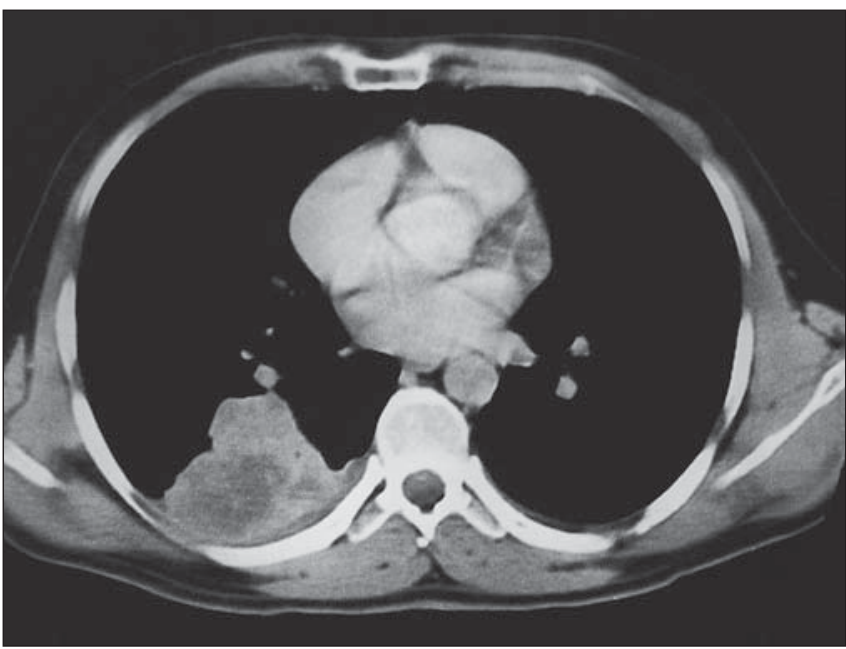

Figura 3. Tomografia computadorizada de tórax com janela para mediastino mostrando massa predominantemente sólida, com impregnação heterogênea pelo meio de contraste, localizada no segmento superior do lobo inferior direito, em amplo contato com a parede torácica adjacente.

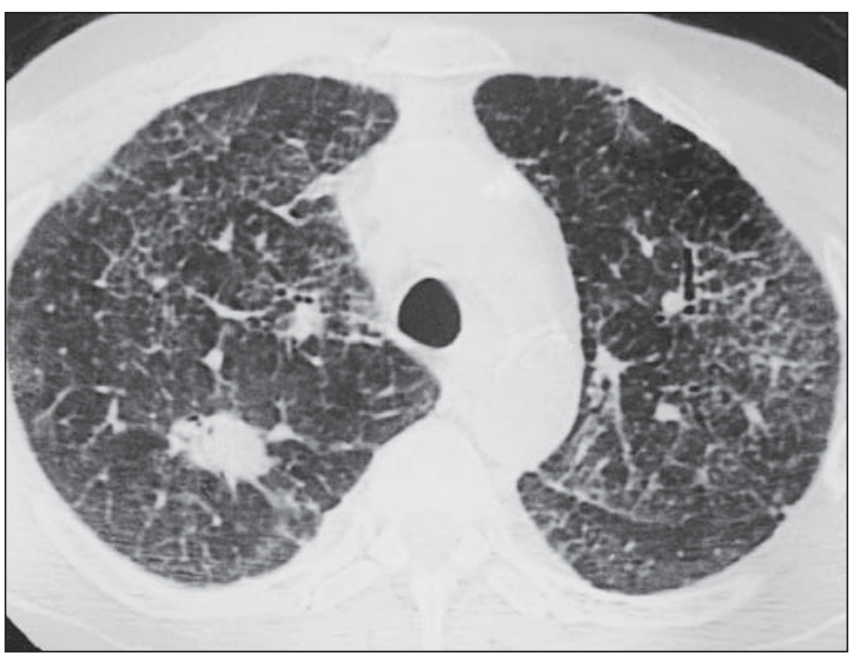

A

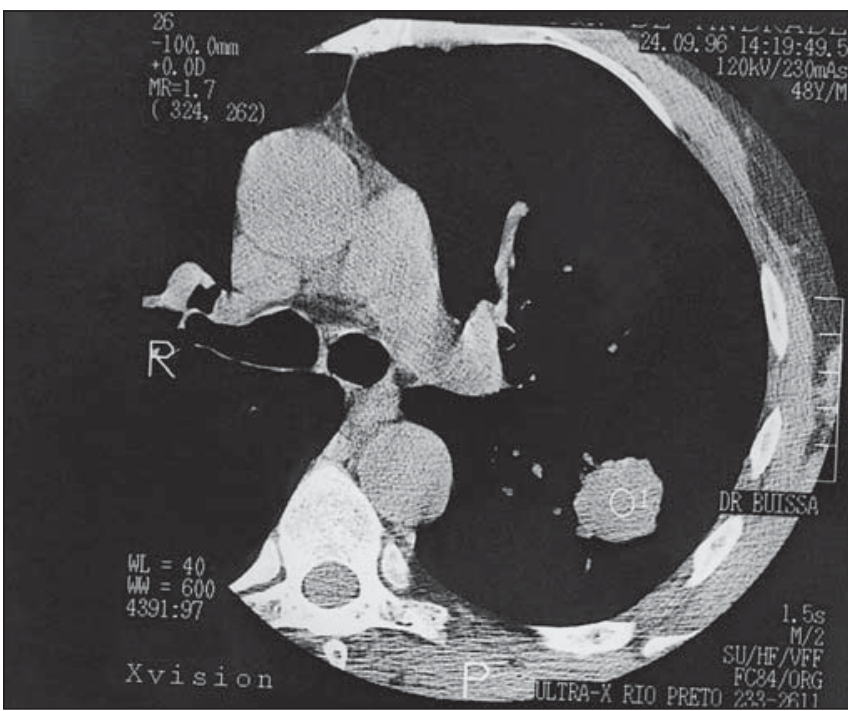

Figura 4. Tomografia computadorizada de tórax com janela para mediastino demonstrando nódulo de contornos irregulares e limites bem definidos localizado no segmento ápico-posterior do lobo superior esquerdo.

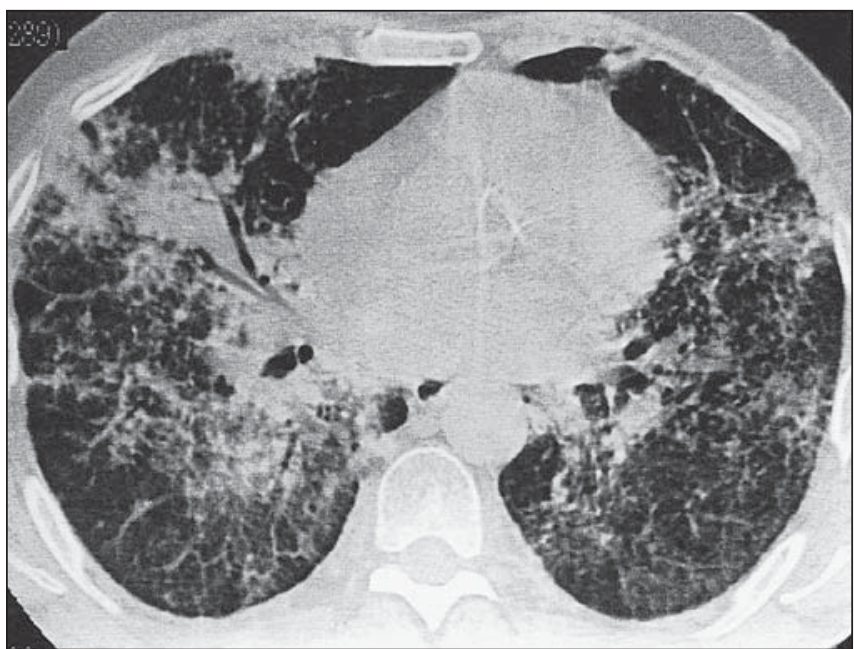

B

Figura 5. Tomografia computadorizada de tórax com janela para parênquima pulmonar evidenciando infiltrado intersticial, com reticulação difusa pelo parênquima, e áreas de consolidação, notadamente na região peri-hilar direita. Observa-se, também, formação nodular no segmento posterior do lobo superior direito.

Ao contrário dos achados descritos na literatura ${ }^{(2,8,10-13)}$, que mostram predomínio do sexo masculino, em nosso estudo não houve predominância quanto ao sexo: $50 \%$ dos casos eram do sexo feminino e $50 \%$ eram do sexo masculino. Kerkering et al. ${ }^{(11)}$ analisaram 41 pacientes, com ou sem imunossupressão, encontrando 28 pacientes do sexo masculino (68,2\%). Chechani e Kamholz ${ }^{(2)}$ avaliaram 48 pacientes com criptococose pulmonar associada à SIDA, sendo $83 \%$ do sexo masculino. Rozenbaum e Gonçalves ${ }^{(\boldsymbol{8})}$, num estudo retros- pectivo de 171 pacientes, encontraram 75,4\% em pacientes do sexo masculino.

A análise das tomografias computadorizadas de tórax evidenciou presença de massa pulmonar com bordas bem definidas e contornos irregulares em nove dos 14 casos de nosso estudo (64,2\%). Observouse que em sete dos nove pacientes com massas pulmonares $(77,7 \%)$ havia íntimo contato destas massas com a superfície pleural, o que não é bem definido nos relatos de literatura. Os nódulos pulmonares representaram o segundo achado mais co- mum, sendo observados em $35,7 \%$ de nossos casos $(\mathrm{n}=5)$; nódulos solitários foram encontrados em dois casos e nódulos múltiplos, em um. Um paciente apresentou associação de nódulo com infiltrado intersticial reticular e em outro houve presença concomitante de massa pulmonar. Doença pulmonar difusa foi evidente em apenas $14,2 \%(\mathrm{n}=2)$.

Khoury et al. ${ }^{(\mathbf{1 3})}$ estudaram 15 pacientes imunodeprimidos e nove imunocompetentes. Nestes, foi verificado o predomínio de nódulos pulmonares (55\%), que apre- 


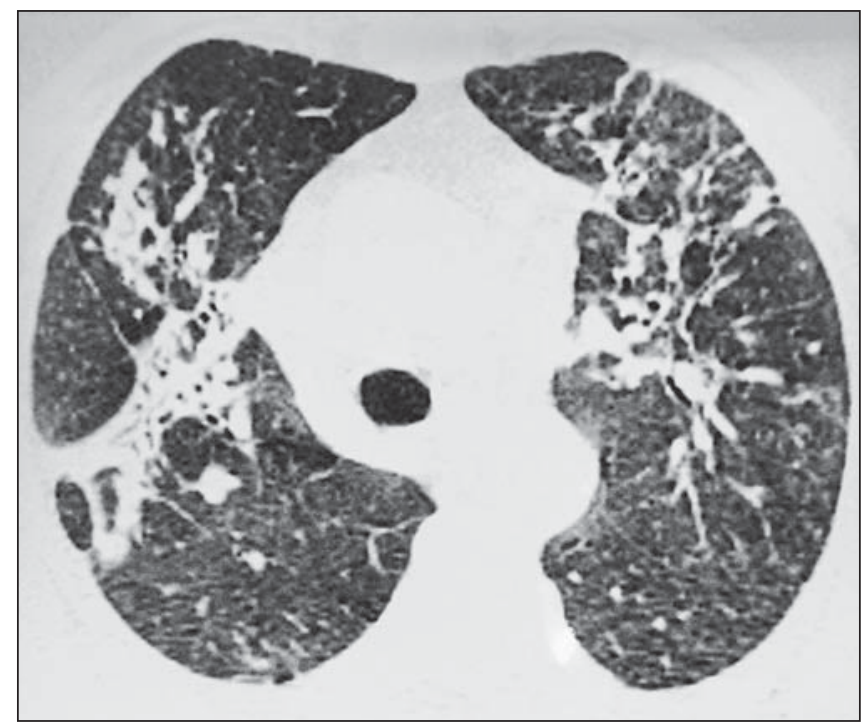

A

Figura 6. Tomografia computadorizada de tórax com janelas para parênquima pulmonar evidenciando áreas de consolidação esparsas em ambos os pulmões, além de espessamento de septos interlobulares e do interstício peribroncovascular.

sentavam diâmetro variando de $0,5 \mathrm{~cm}$ a $4 \mathrm{~cm}$. Roebuck et al. ${ }^{(\mathbf{1 0})}$, em uma avaliação retrospectiva de 44 pacientes HIV negativos, demonstraram a presença de consolidação do espaço aéreo em $34 \%$ dos pacientes e massas pulmonares em $30 \%$. Nakajima et al. ${ }^{(\mathbf{1 4})}$ estudaram dez pacientes com criptococose pulmonar e verificaram a presença de massas pulmonares em $70 \%$ dos casos; o segundo padrão de envolvimento foi o de múltiplos nódulos (20\%). Rozenbaum e Gonçalves ${ }^{(\mathbf{8})}$ referem que os nódulos ou massas pulmonares ocorreram mais freqüentemente em pacientes imunocompetentes, sendo que nos pacientes com SIDA os padrões mais observados foram infiltrado intersticial, infiltrado alveolar, infiltrado misto e derrame pleural. Na nossa casuística apenas dois pacientes, sem imunodeficiência, apresentaram infiltrado intersticial com áreas de confluência, contrariando os achados da literatura, que ressaltam um predomínio deste padrão tomográfico nos pacientes com imunodepressão ${ }^{(7,15,16)}$.

Áreas sugestivas de escavação foram encontradas em dois dos nove pacientes com massa pulmonar $(22,2 \%)$. Os relatos da literatura enfatizam maior freqüência de escavação em pacientes imunodeprimidos, observada em mais de $40 \%$ dos $\operatorname{casos}^{(\mathbf{1})}$.

Em nossa casuística houve predominância das lesões da criptococose nos lobos superiores. Cinco pacientes apresentaram envolvimento isolado de lobo superior $(35,7 \%)$. O lobo médio foi isoladamente afetado em $21,4 \%$ dos casos $(n=3)$ e os lobos inferiores, em 14,2\% $(n=2)$. Dois pacientes $(14,2 \%)$ apresentaram acometimento concomitante dos lobos inferior e superior. $\mathrm{O}$ acometimento difuso dos pulmões esteve presente em 14,2\% dos casos $(\mathrm{n}=2)$. Feigin $^{(\mathbf{1 2})}$ observou, na sua casuística, que as lesões pulmonares da criptococose pulmonar eram mais freqüentes nos lobos inferiores. Jerônimo et al. ${ }^{(7)}$, em um estudo retrospectivo, também demonstraram maior acometimento dos lobos inferiores, presente em cinco dos dez pacientes analisados.

No que se refere à forma de distribuição das lesões, a doença foi unilateral em $78,5 \%$ dos pacientes $(\mathrm{n}=11)$ e bilateral em apenas três $(21,4 \%)$, concordante com os achados da literatura. Feigin ${ }^{(\mathbf{1 2})}$ observou acometimento bilateral em apenas um dos 21 pacientes estudados. Não há definição, nos relatos da literatura, quanto à distribuição segmentar das lesões da criptococose pulmonar. Em nosso estudo houve predomínio das lesões nas regiões anteriores dos pulmões em $42,8 \%$ dos casos $(n=6)$, as regiões posteriores foram acometidas em quatro pacientes $(28,5 \%)$, e ambas as regiões foram comprometidas em $28,5 \%$ dos casos estudados $(n=4)$.
Derrame pleural ou linfonodomegalias não foram encontrados em nosso estudo. Khoury et al. ${ }^{(\mathbf{1 3})}$ demonstraram que a presença de escavação, derrame pleural e aumento linfonodal é limitada aos pacientes imunodeprimidos. Rozenbaum e Gonçalves ${ }^{(8)}$ também evidenciaram áreas de escavação, derrame pleural e envolvimento linfonodal, predominantemente em pacientes com SIDA.

Apenas três dos pacientes do presente estudo $(21,4 \%)$ apresentaram imunossupressão ou doenças concomitantes, contrariando os achados referidos na literatura. Patz e Goodman ${ }^{(\mathbf{1})}$ e Woodring et al. ${ }^{(17)}$ enfatizaram o predomínio da criptococose pulmonar em pacientes imunodeprimidos. Khoury et al. ${ }^{(\mathbf{1 3})}$, num estudo de 24 casos de criptococose pulmonar, demonstraram imunodepressão em $62,5 \%$ dos pacientes estudados. Rozenbaum e Gonçalves ${ }^{(\mathbf{8})}$, em avaliação retrospectiva, relataram deficiência da imunidade em $52,6 \%$ dos pacientes com doença criptocócica.

Em nossa casuística o envolvimento concomitante do sistema nervoso central ocorreu em seis dos pacientes $(42,8 \%)$. No estudo de Chechani e Kamholz ${ }^{(2)}$, dos 29 pacientes com doença disseminada, quatro apresentaram criptococose cerebral e pulmonar. Os 25 pacientes restantes manifestaram envolvimento do sistema nervoso central mais tardiamente. 
A tomografia computadorizada mostrou-se um excelente método para avaliar o grau de comprometimento do parênquima pulmonar na criptococose pulmonar, representando importante etapa para o diagnóstico e para a melhor escolha da conduta terapêutica dos pacientes com criptococose pulmonar.

\section{REFERÊNCIAS}

1. Patz EF Jr, Goodman PC. Pulmonary cryptococcosis. J Thorac Imaging 1992;7:51-5.

2. Chechani V, Kamholz SL. Pulmonary manifestations of disseminated cryptococcosis in patients with AIDS. Chest 1990;98:1060-6.

3. Murata K, Takahashi M, Furukawa A, et al. CT findings of pulmonary infections. Nippon Igaku Hoshasen Gakkai Zasshi 1999;59:371-9. (Abstract).

4. Gordonson J, Birnbaum W, Jacobson G, Sargent
EN. Pulmonary cryptococcosis. Radiology 1974; 112:557-61.

5. Haque AK. Pathology of common pulmonary fungal infections. J Thorac Imaging 1992;7:1-11.

6. Boyars MC, Zwischenberger JB, Cox CS Jr. Clinical manifestations of pulmonary fungal infections. J Thorac Imaging 1992; 7:12-22.

7. Jerônimo ALC, Gonçalves AJR, Correa JC. Lesões pulmonares na criptococose. A propósito de 10 casos. Arq Bras Méd 1986;60:123-8.

8. Rozenbaum R, Gonçalves AJR. Clinical epidemiological study of 171 cases of cryptococcosis. Clin Infect Dis 1994;18:369-80.

9. Miller WT Jr, Edelman JM, Miller WT. Cryptococcal pulmonary infection in patients with AIDS: radiographic appearance. Radiology 1990;175: 725-8.

10. Roebuck DJ, Fisher DA, Currie BJ. Cryptococcosis in HIV negative patients: findings on chest radiography. Thorax 1998;53:554-7.

11. Kerkering TM, Duma RJ, Shadomy S. The evolution of pulmonary cryptococcosis: clinical implica- tions from a study of 41 patients with and without compromising host factors. Ann Intern Med 1981; 94:611-6.

12. Feigin DS. Pulmonary cryptococcosis: radiologicpathologic correlates of its three forms. AJR 1983; 141:1262-72.

13. Khoury MB, Godwin JD, Ravin CE, Gallis HA, Halvorsen RA, Putman CE. Thoracic cryptococcosis: immunologic competence and radiologic appearance. AJR 1984;142:893-6.

14. Nakajima H, Shima T, Usuki N, Fukuda H, Saiwai S, Miyamoto T. CT findings of primary pulmonary cryptococcosis. Nippon Igaku Hoshasen Gakkai Zasshi 1995;55:1032-7. (Abstract).

15. McDonnell JM, Hutchins GM. Pulmonary cryptococcosis. Hum Pathol 1985;16:121-8.

16. Sider L, Westcott MA. Pulmonary manifestations of cryptococcosis in patients with AIDS: CT features. J Thorac Imaging 1994;9:78-84.

17. Woodring JH, Ciporkin G, Lee C, Worm B, Woolley S. Pulmonary cryptococcosis. Semin Roentgenol 1996;31:67-75. 